\title{
A Competence-Based Collaborative Network: The West Midlands Collaborative Commerce Marketplace
}

\author{
Yen Cheung ${ }^{1}$, Helana Scheepers ${ }^{2}$, Mark Swift ${ }^{3}$, Vincent Lee ${ }^{1}$, and Jay Bal ${ }^{3}$ \\ ${ }^{1}$ Clayton School of IT, Monash University, Victoria 3800, Australia \\ ${ }^{2}$ Swinburne University, Victoria 3122, Australia \\ ${ }^{3}$ WMG, University of Warwick, Coventry, CV4 7AL, UK \\ ycheung@infotech.monash.edu.au, HSCHEEPERS@groupwise.swin.edu.au, \\ Mark.Swift@warwick.ac.uk, Jay.Bal@warwick.ac.uk, \\ vincent.lee@infotech.monash.edu.au
}

\begin{abstract}
We present a competence-based collaborative network, the West Midlands Collaborative Commerce Marketplace (WMCCM) that was successfully employed to gain new business opportunities for SMEs in the West Midlands region of the UK. The competence profiling methodology employed places an emphasis on what SMEs are capable of doing rather than what products they make. This generates an increased number and wider range of enquiries to the SME. Further, a combination of tender feeds classified by semantic analysis of the tender requirements matched to the SME capability with a novel partnership formation function generates higher level systems type opportunities for these SMEs, which they would have been unable to undertake on their own. The paper discusses the functionality of the WMCCM using a case study to demonstrate its functionalities with reference to the I-BPM framework from the ECOLEAD project. It also highlights the roles in managing competence exchanges in collaborative networks as well as providing a common infrastructure for businesses to launch and operate their virtual organizations.
\end{abstract}

Keywords: collaborative network, competence, profiling, tender.

\section{Introduction}

The introduction of Internet technology and electronic communication in the mid 1990s, have created expectations about how businesses could benefit from this new electronic environment i.e. electronic marketplaces (eMarketplaces) and how they could have access to global economy [1]. However despite rapid developments and implementation of eMarketplaces, there is only a modest uptake of this technology with many cases of failure to deliver promises being reported in the literature [2]. Hence to date, there has been a limited acceptance of eMarketplace models that has given investors confidence in their long-term financial viability as independent businesses. The main reason for such failures is the lack of robust models and understanding in the structure and complexities of interactions (or collaborations) amongst the various entities in eMarketplaces. Although collaborations bring huge benefits for the 
stakeholders, a high failure rate among collaborative organisations is reported in the literature [3]. In the case of eMarketplaces, both benefits of collaboration and the potential of the Internet should lead to even more benefits for organisations. However as discussed above, this is not the case as their development and deployment has been rather ad hoc. Indeed various projects around the world (e.g. ALIVE, GENESIS, ATHENA and ECOLEAD to name a few), are working to provide solutions to the challenges of the emerging discipline of collaborative networks of which eMarketplaces are an example.

Triggered by opportunities provided by eMarketplaces, distributed global eBusiness collaborations have given rise to virtual enterprises (VEs), where autonomous organisations collaborate primarily over the Internet to achieve a common goal. These VEs are said to operate in collaborative-networked breeding environments. This area of research in Internet enabled collaborative systems is generally known as collaborative networks (CNs) [4]. For example, in Europe, ECOLEAD (European Collaborative networked Organizations LEADership Initiative) addresses the areas of CNs such as a framework to form the basis for dynamic and sustainable-networked organisations.

In this paper, a practical and successful example of a virtual organisation breeding environment (VBE), the WMCCM is presented as a competence-based collaborative network. The design and functionalities of the WMCCM is described using the reference framework of ECOLEAD.

The structure of the paper is as follows: in the following section we provide background on $\mathrm{CN}$ frameworks as suggested by ECOLEAD, followed by a description of the West Midlands Collaborative Marketplace (WMCCM), its design and functionalities such as the competence profiling methodology. This is followed by a section presenting the outcomes of the system i.e. providing improved access to existing and new market opportunities as well as enhancing collaboration amongst stakeholders to generate new opportunities. In the conclusion we summarise our findings and articulate some directions for further research.

\section{Collaborative Networks}

A comprehensive $\mathrm{CN}$ framework called the Integral business process management framework (I-BPM) has been developed by Romero and Molina [5] based on the ECOLEAD project [4]. The I-BPM takes a holistic view of CNs and includes the creation as well as ongoing management of virtual organisations (VOs) and virtual breeding environments (VBEs). A VBE is a pool of organisations and their related supporting institutions that have both the potential and interest to cooperate [6]. A subset of this pool is a VO that can be formed on a short term (a dynamic virtual organisation) or long term (a permanent structure such as a cluster) basis.

The I-BPM consists of the following five process groups:

- VBE actor management - creation and update of information about members, VBE structure and member competence profiles;

- VO creation - identification of business opportunities, initial VO planning, partner search and the development of partner agreement;

- VO Management - management of VOs from their initialization through to their demise; 
- VBE General management - management of the overall activities of the VBE for example strategic and marketing management and ICT management;

- $\quad$ VBE horizontal management - management of the processes that cuts across the VBE such as trust management, performance management and innovation management.

The following section presents the design and functionalities of the WMCCM as a CN that includes some of the major processes of the I-BPM framework.

\section{The WMCCM}

The goal of the WMCCM was to address a critical need of SMEs in the West Midlands. Like the rest of the UK, the West Midlands has a large number of SMEs. In the UK, overall SMEs make up the majority of businesses with a total of 4.3 million enterprises employing 22 million people [7]. Their inherent size means that many operate within limited financial resources, skills and competences resulting in limited economies of scale and scope and an inability to react quickly to these pressures and demands [8]. West Midlands UK research however showed that for SMEs supplying the automotive sector, on average just under $50 \%$ of their turnover is derived from that sector [9]. It would therefore be impractical for a small business firm to sign up to these vertical online e-marketplaces, each with its own unique protocols, procedures, practices and ultimately costs. To participate in e-commerce via the many eMarketplaces that exist is not viable for SMEs with limited funds and technical capability.

The WMCCM is a specific case study to test and validate the regional marketplace model. It is a six-year, €5.2 million regional ICT initiative, funded under the European Regional Development Fund to support the economic and social conversion of industrial areas that face structural difficulties.

\subsection{WMCCM Workflow}

WMCCM is designed to support the following key workflow processes as shown in Figure 1. The workflow, as illustrated from left to right in Figure 1, are described below:

1. Sourcing new business from varied e-tender external online sources including a range of both public and private sector contracts. Firms can also generate tenders for their own needs.

2. Competence Profiling of SMEs. This is a structured process that measures capability within firms using a standardised formal questionnaire with the objective of generating trust and giving easily comparable information. The focus of the questionnaire is to discover what the firm can do, rather than what they are doing currently. The competence profile also captures a range of hard and soft factors so that firms with similar cultures can be identified when trying to form effective collaborations and virtual organisations. The competence profile of each company is indexed against a common system ontology.

3. The classification of tender requirements and SME capability uses the same common ontology. The daily tenders listing each day (200+ tenders) is semantically analysed and the tender requirements are automatically classified by 


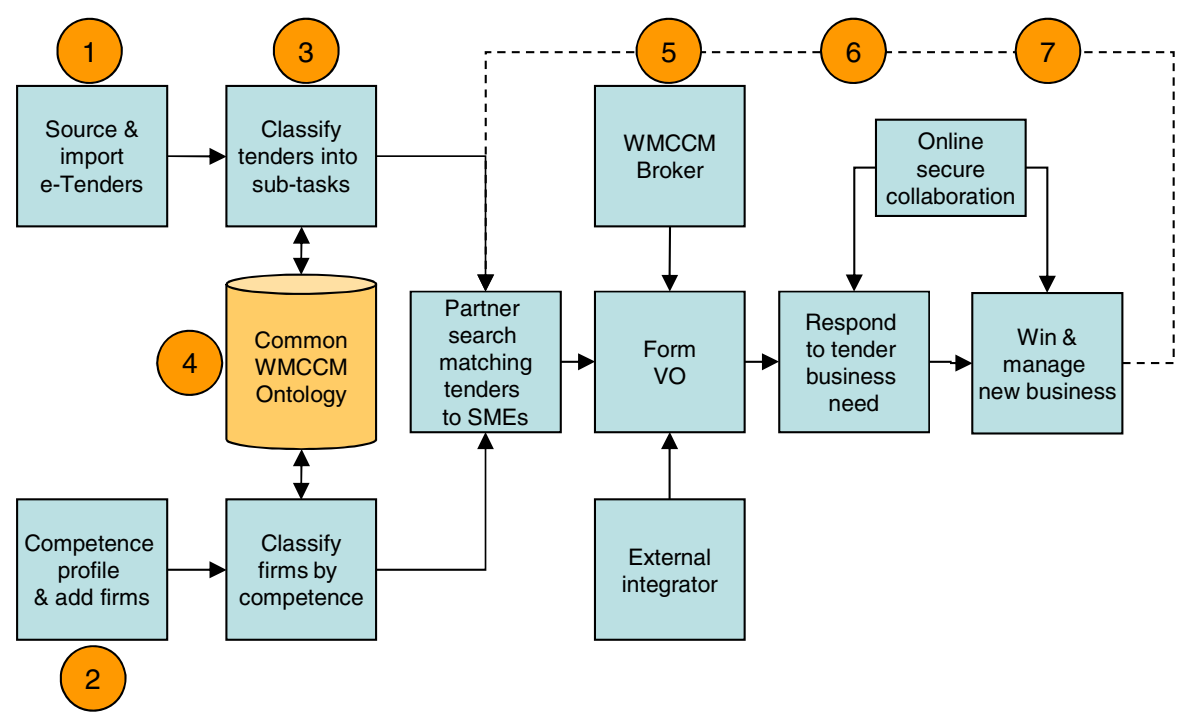

Fig. 1. The WMCCM workflow

process capability required, skill need, location and quality standards by the WMCCM system.

4. Match tender to individual or group of SMEs using WMCCM online Partner Search System. The Partnership Search system is used when a tender requires a competence, which SMEs cannot fully cater for. This is a common situation since the tenders are higher value "systems" type tenders. It is a two-stage search process. The first stage matches against an absolute need or hard factors such a capability in networks, injection moulding, metal spinning etc. The second stage is a preference or soft factor scoring process to generate a partnership which has common cultural elements and is more likely to be able to work together. These relate to industry knowledge, size, quality standards, location etc. The system scores marks for each eligible company and then suggests a best-fit partnership based on the highest matched scores. The SME's are automatically alerted by a daily email giving detail about the tender opportunity.

5. The results of the partner search suggest an initial best fit grouping of SMEs who collectively have the potential capability to bid for the tender contract. At this stage the VO starts to take shape as members undergo a stage of team forming through a number of initial face-to-face meetings. WMCCM provides secure on-line collaboration spaces to facilitate this.

6. To respond to tender (business need) and collaborate online in secure project spaces. Default functionality includes document storage, simple project and task allocation management and discussion forums with associated knowledge management tools.

7. If the bid is successful and the contract won, further collaboration tools can be used such as an integrated supply chain visibility facility.

In summary, rather than just measuring current operational capability, competence profiling seeks to determine transferable capability. SMEs who are competence profiled 
are matched against a dynamic ontology, which allows their capabilities to be matched against the tasks of any online tender. This is the matching of tasks (tender requests) with resources (the SMEs capability) with the view of stimulating innovation.

As of early February 2010, the WMCCM had over 9,000 registered SME organisations, covering a broad range of industrial competence. Over 350 of WMCCM SME members have been competence profiled. The following section provides the SME Military Tender case study that was a result of a competitive tender procurement invitation issued by a department of the UK MoD, Defence Scientific Technology Laboratories (DSTL) in April 2006. The goal of the tender is to supply complex and varied engineering services for ten years.

\subsection{A Case Study: SME Military Tender}

The DSTL tender was an opportunity to apply automotive industry best practice to form a VO, comprising of multi-disciplinary SMEs in order to meet future DSTL engineering requirements. Due to the scale and complex nature of the DSTL technical requirements and scale of the contract value at over $€ 15 \mathrm{~m}$, it was accepted that a typical engineering SME within the West Midlands, UK could not provide the wide range of competencies nor realistically expect to navigate the intricate tendering procedures independently.

Thus a collaborative VO was established through utilising the WMCCM to address the specific requirements of the DSTL contract. Aspîre Consulting, a Defence based SME and WMCCM member, was used to act as a conduit between the automotive and military industry sectors, to facilitate the long and intricate DSTL procurement process and in the future to act as a potential prime contractor.

When the DSTL tender was received by WMCCM, it was automatically processed by the in-built dynamic ontology, which splits the tender into a number of sub-tasks including industrial market, technical skill and geographic location.

The online partner search selection tool was run to identify a short list of potential consortium members covering each area of technical expertise. This matching of capability is a key part of grouping potential members, in order to build a dynamic VO. Each company was then contacted and invited to form part of the collaborative consortia, attending a number of face-to-face briefing sessions. The VO structure of the DSTL bid can be seen in Figure 2.

The collaborative bid aimed to provide DSTL with a "one-stop-shop" for all of the engineering service disciplines detailed in its competitive tender call. In addition to the membership of WMCCM and its approach seeks to give a number of further benefits:

- The ability to address all of DSTL's needs both from a capability and capacity perspective without any additional investment in tooling, machinery and software.

- The ability to offer additional services from other SME members of WMCCM to meet DSTL additional service options. WMCCM has a wider membership of 4,000 SMEs, each with a brief business or detailed competence profile. Consequently if the combined capability and capacity of the companies identified in this proposal are unable to meet the task requirements set by DSTL then within a short time period an alternative supplier can be identified from the WMCCM membership to join the team. 


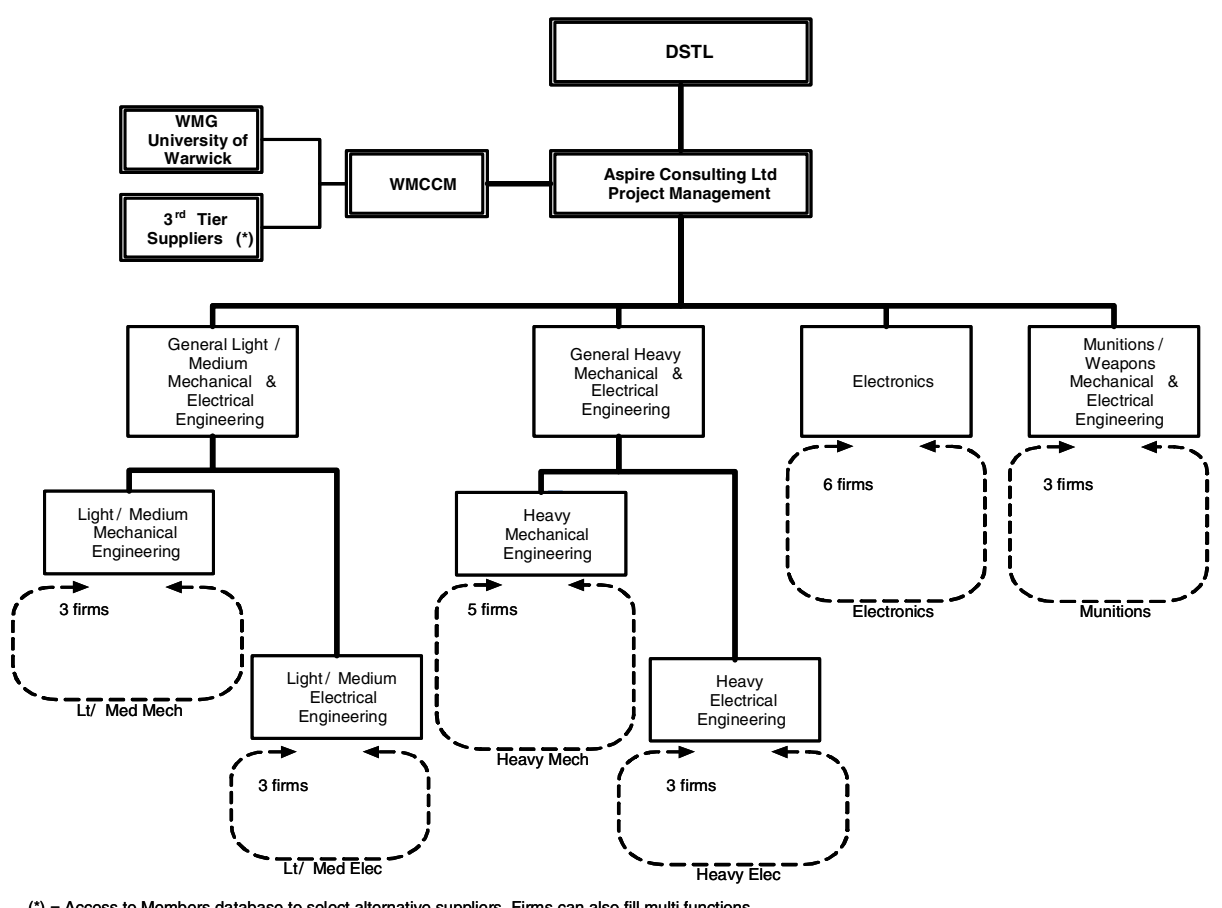

$(*)=$ Access to Members database to select alternative suppliers. Firms can also fill multi functions

Fig. 2. Proposed DSTL response VO structure

\section{Discussion}

WMCCM incorporates aspects of the business process groups of the I-BPM framework such as VBE actor management, VO creation and limited aspects of VBE horizontal management. The workflow of the WMCCM as shown in Figure 1 will be compared with the business processes of the I-BPM framework as shown in Table 1.

The competence profile of SMEs is performed by the WMCCM team, which visits those SMEs that wish to be competence profiled to extract their capabilities (such as unique metal turning capability). The identified capabilities are classified using the common ontology (process 4 in Figure 1) within the WMCCM. The conduct of the competence profiling is process 2 as shown in Figure 1. Companies that do not wish to be competence profiled can simply register with the WMCCM to use the features of the system such as browsing through the marketplace or search for partners to form teams for collaborative projects. The main difference between profiled and non- profiled members is that profiled members have a higher visibility on the Internet in terms of their capabilities. So far, successful cases in the WMCCM mainly came from those SMEs that have been competence profiled. SMEs when participating in a $\mathrm{CN}$ organise the projects themselves and hence, no functionality exist in WMCCM to identify and manage the roles of VO. Online collaborative project spaces are available within the WMCCM should the teams decide to use this facility (processes 5, 6 and 7). However processes 1 and 3, initiates collaboration opportunities for members through the matching of tenders to individuals or groups. The system functions like a 
Table 1. Comparison between I-BPM processes and WMCCM processes

\begin{tabular}{|l|l|}
\hline I-BPM framework process & WMCCM process \\
\hline VBE Actor Management & Competence Profiling
\end{tabular}

'match-making' facility linking capabilities with requirements and any agreement or contract negotiations between partners are handled outside the WMCCM system.

A factor that distinguishes WMCCM from other proprietary $\mathrm{CN}$ systems is that the developers of the WMCCM operate in a trust worthy environment. Experts within the Warwick Manufacturing Group (WMG) at the University of Warwick (UK) capture competence profiles and WMG is seen as a respected impartial organisation. The WMCCM provide a trustworthy environment within which activities takes place.

\section{Conclusion}

WMCCM is a free market model, empowering and encouraging SMEs to dynamically form VOs driven by business needs from the prospect of gaining new work from online tender requests. It is one of the first empirical examples of a VBE for SMEs. WMCCM is a practical implementation of the VO creation framework of the I-BPM framework. At the same time, it also supports other business processes of the I-BPM framework. Further work involves expanding the model to link up other regions of the world such as Australia, which has a similar SME profile.

\section{References}

1. Mehler, M.J., Worthington, R., Fife, E., Pereira, F.: The Internet a la Fin de Siecle: The prospect for Internet commerce Centre for telecommunications management. University of Southern California, Los Angeles (1997) 
2. Fisher, J., Craig, A.: Developing business community portals for SMEs: Issues of design, development and sustainability. Electronic Markets 15(2), 136-145 (2005)

3. Chituc, C.M., Azevedo, A., Toscano, C.: A Framework Proposal for Seamless Interoperability in a Collaborative-Networked Environment. Computers in Industry 60, 317-338 (2009)

4. Camarinha-Matos, L.M., Afsarmanesh, H.: Virtual Enterprises and Collaborative Networks. In: 18th IFIP World Computer Congress, pp. 3-16. Kluwer Academic Publisher, Dordrecht (2004)

5. Romero, D., Molina, A.: VO breeding environments \& Virtual Organisations in Integral Business Process Management Framework. Inf. Syst. Front 11, 569-597 (2009)

6. Kürümlüoglu, M., NØstal, R., Karvonen, I.: Base Concepts. In: Camarinha-Matos, L., Luis, M. (eds.) Virtual Organisations: System and Practices, pp. 11-28 (2005)

7. National Statistics, Press release, Small Business Service website, http://ww.sbs. gov.uk/SBS_Gov_files/researchandstats/SMEstats2005pr.pdf

8. Sunday Times: Skills shortage holding back e-business innovation says SMEs, Sunday Times, February 1 (2000)

9. Szczygiel, M.: AutoLean II - Project. Summary Report, Accelerate Partnership (November 2000) 